\title{
Measurement Methods to Analyze Changes in Coordination During Motor Learning from a Non-linear Perspective
}

\author{
Robert Rein* \\ Department of Neurology Institute of Health Promotion and Clinical Movement Science German Sport University \\ Cologne, Am Sportpark Müngersdorf 6 Cologne, Germany
}

\begin{abstract}
During the last two decades investigations into motor learning have gone beyond the traditional discrete summary statistics and more and more complex process oriented movement variables are being investigated. This increase in the complexity of data entails also an increase in the complexity of the data analysis. The present paper serves as an introduction for sports scientists to several different analysis methods, which have produced many interesting insights in the area of motor control and motor learning over the last few years, thereby highlighting non-linear aspects of motor learning. An approachable introduction to root-mean square measures, uncontrolled manifold analysis, principal component analysis, and cluster analysis is given. These analysis tools enable sports scientists to investigate motor learning from a non-linear perspective and to gain a better knowledge of the processes occurring during motor learning.
\end{abstract}

Keywords: Movement variability, motor coordination, statistics.

\section{INTRODUCTION}

For quite some time the focus of studies in motor learning was set on changes in performance variables. Accordingly, a large body of literature describing how specific performance variables change over time during learning is available [1-3]. For example, reaction time being one of the most prominent performance variables has been studied using all possible permutations of tasks and conditions and many interesting insights have been gained by this line of research $[3,4]$. The types of variables used in these studies can be characterized as discrete summary statistics as they collapse a complex phenomenon like an action onto a single number and this approach has been used extensively in the area of movement studies as well. For example a javelin throw is represented through the throwing distance [5]. One problem with these discrete summary statistics being obviously that much information is lost during the analysis [6]. In Fig. (1) two curves with equal mean and standard deviation but obviously representing quite different waveforms are depicted. Thus, if these waveforms would be to represent joint angle curves, two quite different movements would result despite equal summary statistics.

Over the last two decades the focus in studies of learning has somewhat shifted and more and more studies, not only investigating performance variables but also the actual process generating the performance, have emerged as their importance has been noted in the literature [7]. The theory of dynamical systems to motor control (DST) has proven as a particular fruitful framework for investigating the changes

*Address correspondence to this author at the Department of Neurology, Institute of Health Promotion and Clinical Movement Science, German Sport University Cologne, Am Sportpark Müngersdorf 6 Cologne, Germany; Tel: +49 (0)221 4982 7290; Fax: +49 (0)221 4971 726;

E-mail: r.rein@dshs-koeln.de

\# occurring during motor learning emphasizing non-linear behavior $[8,9]$. Investigations under this paradigm focus on global patterns of actions, so-called attractors (collective variables) governing behavior $[9,10]$. This let to led also to a shift of the research focus. From a DST perspective the actual process generating an action becomes much more central for the investigation $[7,8,10-14]$. Research under this paradigm has shown, that there is not a one-to-one mapping between movement patterns underlying an action and the resulting outcome of the action [15], thereby emphasizing

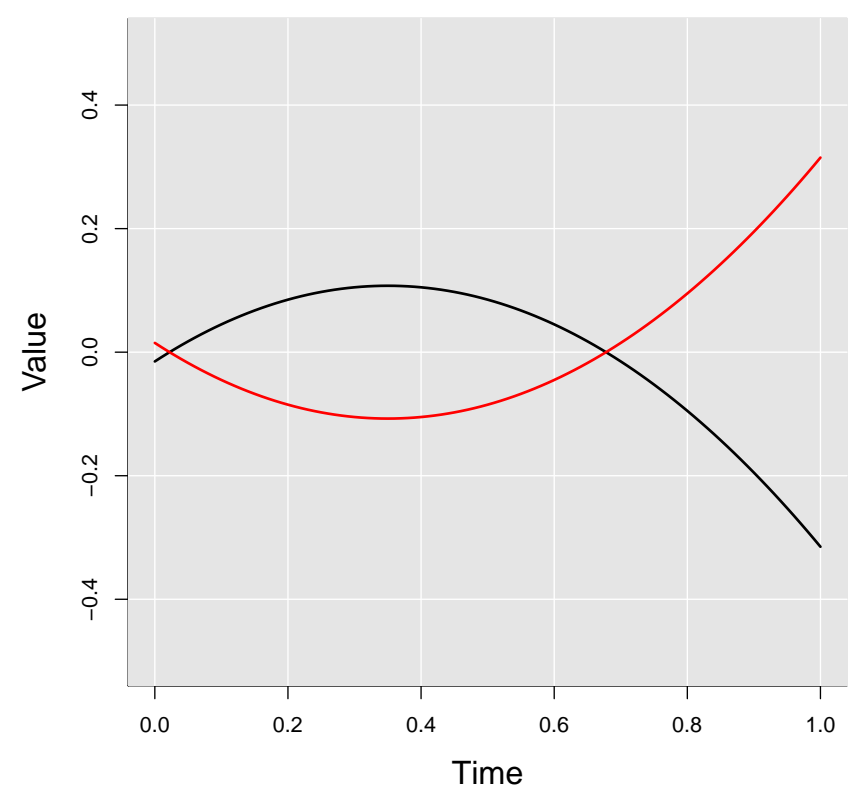

Fig. (1). Two waveforms with equal mean, range and standard deviations (Time and Value are of arbitrary units). 
the analysis of process-oriented variables [16]. Thus, in order to discover the non-linear dynamics underlying movement a change of the research focus is necessary. With this shift comes also a change in the type of variables being investigated and often the dependent variables used in these kinds of studies are rather of continuous and more complex nature as they describe processes in time. For example, the changes in joint segment angles during the execution of a javelin throw over a learning period. This entails not only a more refined view of what is actually happening during learning but also means that the analysis becomes much more involved as the data structures being analyzed are much more complicated compared to the more straightforward discrete summary statistics. Thus, a researcher investigating continuous data must have a somewhat better understanding of data analysis techniques compared to for example a straightforward reaction time measure [17]. The present paper addresses this problem and provides some guidelines about new research methods tailored to this kind of datasets thereby serving as an introduction for sports sciences researchers interested in motor learning from a non-linear perspective. Several different measurement techniques useful for process-oriented variables will be presented. In the following, root-mean square approaches, Principal component analysis, Uncontrolled manifold analysis, and cluster analysis will be introduced. The explanations of the methods thereby follows mainly the same structure, where first the motivation for the method is stated, followed by an introduction of its execution, some examples in the literature, and concludes with a reference to an article which is suitable as a first starting point for the interested reader. Central to the following approaches, is that in one way or another they are all concerned with the degrees of freedom problem to motor control $[8,18-20]$. How actors choose a particular solution from the infinite many available is still one of the great problems in motor control and motor learning and therefore it comes as no surprise that many methods circle around this phenomenon. For sports scientists this problem is of particu- lar interest as they deal with actions, which are inherently more complex than many tasks used in the laboratory [21].

\section{ROOT MEAN SQUARE APPROACHES}

Root mean square approaches have been used with continuous data for quite some time but have been recently extended into new directions. The basic question behind these approaches is to measure the similarity between different signal waveforms. Thereby, root-mean square (RMSE) and normalized root-mean square (NoRMS) measure the similarity between two waveforms whereas the Cauchy criterion compares several waveforms together.

One of the more traditional approaches comparing the analysis of movement patterns based on time continuous data is the root-mean-squared-error (RMSE) criterion [1]. In its simplest formulation the deviations between two curves (e.g. a trial pathway and a criterion pathway) at successive time points of a trial pathway are calculated, squared, summed, and the square root is taken from the resulting deviation score. In Fig. (2) two synthetic pathways are depicted. In reality these could be for example joint angle or position data. The RMSE criterion calculates the differences between the two curves.

Basically, the procedure calculates the Euclidean distance between two vectors in $\mathrm{R}^{\mathrm{N}}$, where $\mathrm{N}$ refers to the number of time slices of the two curves.

$$
R M S E=\sqrt{\sum_{n=1}^{N}\left(x_{i n}-x_{c n}\right)^{2}}
$$

$\mathrm{N}$ is the number of time frames, $\mathrm{i}$ the trial, and $\mathrm{c}$ is the criterion trial.

A condition inherent to this approach comprises that both curves must have the same number of time points otherwise a comparison is not possible. Thus, in most application the data is time warped such that both curves posses the same number of frames. One problem with this approach is that

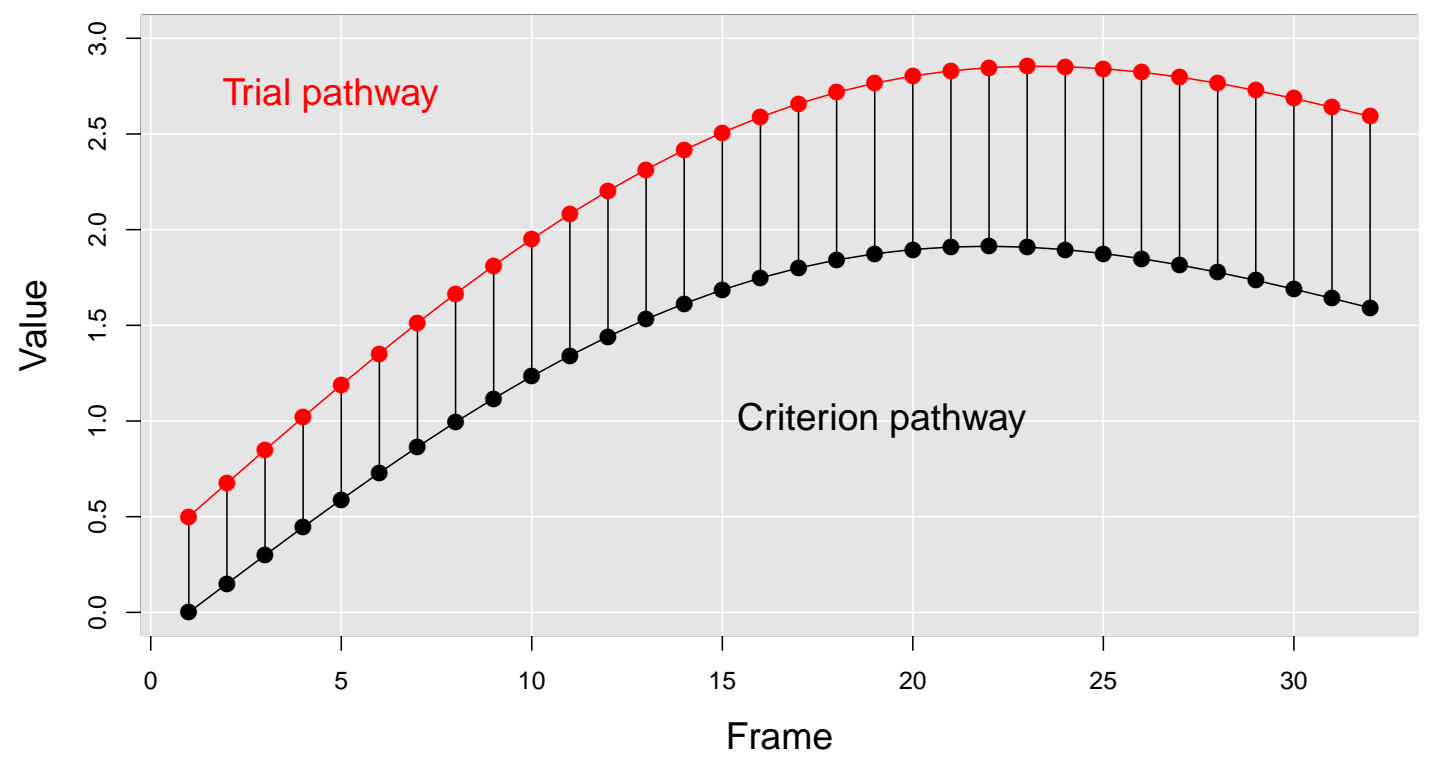

Fig. (2). RMS differences between two trial pathways. 
the magnitude of the difference varies with the number of time points used [compare also 22]. Thus, when comparing across participants the RMSE criterion should be normalized for example by dividing the score with the number of time points.

Sidaway, Heise, and Zohdi [23] developed an extension of this approach which estimates the variability in coordination between two joint angles based on angle-angle plot diagrams. Angle-Angle plot diagrams provide a simple approach to depict the coordination between body segments and are often used only heuristically. The procedure was called Normalized-Root-Mean-Square (NoRMS) method and measures the deviation of a group of trials from an average trial. The desired angle kinematics of two angles are time normalized in order to obtain the same number of time slices for each trial. The underlying assumption therefore is that movements are scaled in a similar fashion and the relative placement of key events does not change between trials. Based on the time-normalized representation of the group of trials, a mean trial is calculated and subsequently the deviation from the mean trial for each trial using the RMSE for each angle is calculated (similar to the standard deviation in univariate statistics). The procedure was identified by Mullineaux, Barlett, and Bennett [24] as suitable to identify consistency in non-linear movement patterns. Although derived from angle-angle plots the procedure is not confined to angular data only but could also be used with positional or similar data.

In Fig. (3), a synthetic angle-angle plot is shown. Individual trials are depicted in dark gray and the mean trial is shown in black. The NoRMS score calculates the deviation of the individual trials from the mean trial. As two angle are used, two variation scores per trial are obtained which are squared, summed and the square root is taken yielding a single score. The resulting NoRMS score is scaled according to the number of data frames $\mathrm{N}$ and the excursion of the mean curves.

$$
\begin{aligned}
& R_{i n}=\sqrt{\left(x_{1 n}-\bar{x}_{1 n}\right)^{2}+\left(x_{2 n}-\bar{x}_{2 n}\right)^{2}} \\
& R M S_{i}=\sqrt{\frac{\sum_{n=1}^{N} R_{i n}^{2}}{N}} \\
& N o R M S=\frac{\sqrt{\sum_{i=1}^{I} R M S_{i}}}{I^{*} L}
\end{aligned}
$$

$\mathrm{I}$ is the number of Trials, $\mathrm{n}$ is the frame number and $\mathrm{N}$ is the total number of frames, and $\mathrm{L}$ is the resultant excursion of the mean angle waveform.

Sidaway and colleagues [23] applied the method to data from a rhythmical ski simulator task. The results indicated that skilled performers show greater consistency in movement execution compared to novice performers in the kneeknee and hip-knee angle-angle plots. Hodges, Hays, Horn, and Williams [25] applied the procedure to skill acquisition data from a novice player in a soccer in-step movement. The results showed decreasing NoRMS scores throughout the learning period indicating smaller movement variability in the lower limb segments $[25,26]$. The NoRMS procedure has also been applied to gait data [27-29].

A procedure similar to NoRMS was used in a the study conducted by Chen, Liu, and Mayer-Kress [30] investigating motor learning in a pedalo locomotor task. A variation of the RMS-approaches called the Cauchy criterion was used. The Cauchy criterion measures the differences between movement pattern performed on consecutive trials. The criterion was based on the differences between the spatial joint displacements in 3D space using a time-normalized representation of the movement. Differences between movement trajectories are squared and summed over all variables. From this measure the square root is taken and divided by the

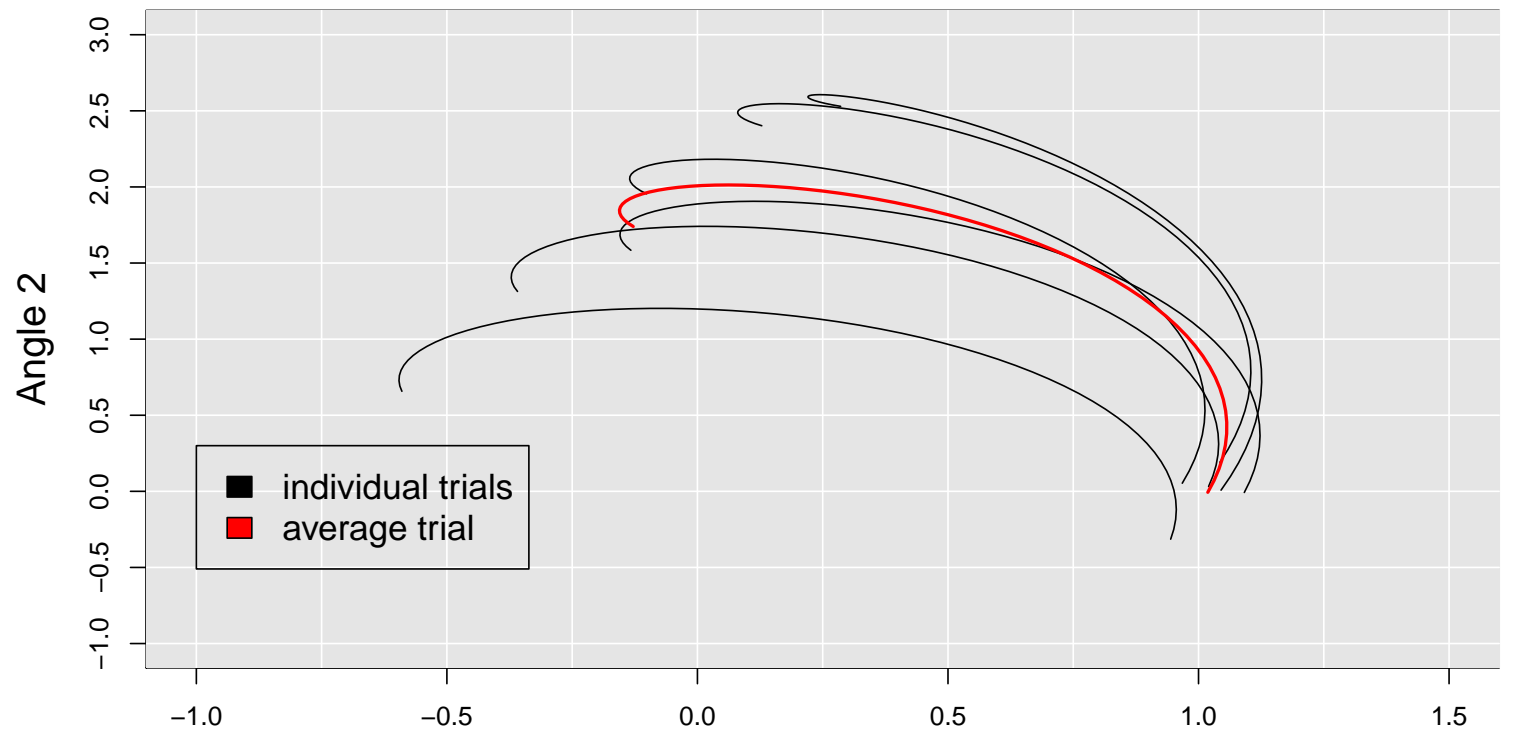

Angle 1

Fig. (3). Angle-Angle plots of individual and mean trial. 
number of trials I - 1 and number of input variables $\mathrm{K}$.

$$
C_{i}=\frac{1}{K(I-1)} \sum_{n=1}^{N} \sqrt{\sum_{k=1}^{K}\left(x_{k n(i+1)}-x_{k n i}\right.}
$$

Hence, when the movement trajectories in subsequent trials are similar to each other, a small $\mathrm{C}_{\mathrm{i}}$ value is obtained. Applied to the pedalor locomotor task the results indicated a decrease of the Cauchy scores during the learning period [30]. The rationale of the approach is based on the assumption that throughout learning the variation of movement trajectories should decrease and the movement patterns of the performers converge onto a certain class of individual patterns.

In summary, the three approaches presented here all apply root-mean square approach to estimate deviations between executed trials. The Cauchy criterion estimates the differences between two trials, whereas the NoRMS procedure estimates the variability of several trials together. The basic procedures hold few assumptions regarding the distributions of the raw data and only penalizes deviations greater in magnitude than smaller deviations by squaring the deviations, which seems to be an intuitively valid assumption. By using a whole body representation in the Cauchy-score differences in movement coordination all body segments included can be assessed simultaneously.

Important for all procedures is the assumption, that time normalization of trials does not influence the variability of the signal and accordingly biases the deviation scores [22, $31,32]$. A potential confounding factor might be the introduction of the range normalization used in the NoRMS procedure. When the scores are range normalized the underlying assumptions is that small deviations in one angle with only small range of motions are more important compared to deviations in another angle possessing a greater range of motion as the range normalization imposes a weighting scheme on the signal waveforms. This assumption might not be universally valid since one can argue that for the CNS, limiting the range of motion in one angle represents an equal difficult control problem as moving a segment. For example, when an actor waves an arm and holds the elbow angle constant, the elbow angle has to be specifically controlled by the CNS due to the changing interaction torques resulting from the arm motions [33]. Thus, range normalization might mask this information and potentially bias the results [34, 35]. A problem in particular with the NoRMS procedure is related to the calculation of an average trial as the mean is quite sensitive to outliers. Such outlier trials will increase the deviation score disproportionately and potentially yield incorrect conclusions. Similarly, if the kinematics exhibit clustering behavior where the investigated group of trials contains sub groupings, the level of variability within each group may be very small but because of the usage of an average trial, the dissimilarity scores are potentially inflated.

\section{PRINCIPAL COMPONENT ANALYSIS}

Another analytical tool suitable for analyzing changes in movement coordination during motor learning is principal component analysis (PCA) [17, 36, 37], which is in particular helpful for investigating actions involving many degrees of freedom. Principal component analysis is also called Karhunen-Loève expansion or singular value decomposition [17]. Often used as a compression routine for example in computer science, principal component analysis allows to reduce the number of dimensions needed to describe a multidimensional process [38]. The motivation for principal component analysis is the question whether it is possible to represent a complex dataset containing many variables only through a few "principal" variables containing the main information of the data. The set of principal components represent orthogonal components of the variation in the data and are ordered according to the magnitude of the variation [39]. The first principal component explains the largest variance, the second component the second largest variance in the data and so on. If there are linear relationships between the different input variables these procedure yields typically only few principal components, which explain the bulk of the variation in the data.

The calculation of principal components is based on an orthonormal linear transformation of a data vector $\mathrm{x}$, containing several variables, into uncorrelated principal components $z=A^{\prime} x$, where $A$ is an orthogonal matrix [38]. As one seeks to compress the information in the data onto fewer dimensions, an intuitive approach seeks to explain the largest variance in the data through a combination of orthogonal and thus uncorrelated components. It can be shown that the solution to this optimization problem is given by the eigenvalues and corresponding eigenvectors of the sample covariance matrix of the dataset $[17,38,40]$. Accordingly, by using only the first few principal components one obtains a compressed representation of the input space. The contribution of each original variable to the principal component is described by the factor loadings or coefficients, which are simply the eigenvectors of the sample variance-covariance matrix. Geometrical, the principal components form a new rotated coordinate system, which is rotated such to maximize the variance along the axes. The new rotated coordinates of the data are called scores and describe the original data using the principal components $[38,41]$. Studies using PCA typically are interested in the number of components necessary to describe the data and what their individual magnitudes are. Sometimes also the factor loading is investigated to gain information about which variables contribute to individual principal components [40] or the direction of the eigenvectors is analyzed [42]. An interesting interpretation of the eigenvectors is given by Haken [43, p.158]. As the principal components describe the global behavior of the system, in some cases they can be interpreted as the order parameters of the system. Therefore, the eigenvectors may provide a direct interpretation based on a non-linear perspective [see also 37].

In Fig. (4a) joint angle data from a single participant performing a hook shoot is shown. The raw data matrix contains 10 variables. Purely, by visual inspection one can already see that there are some correlations between the different joint angles curves as the shapes of the waveforms show some strong similarities. Using a principal component analysis two principal components were extracted. These two components explain already more then $90 \%$ of the variation in the data as depicted in Fig. (4b). In Fig. (4c) the waveforms for these two principal components are shown. The first principal component (PC1) depicts a falling wave- 
a)

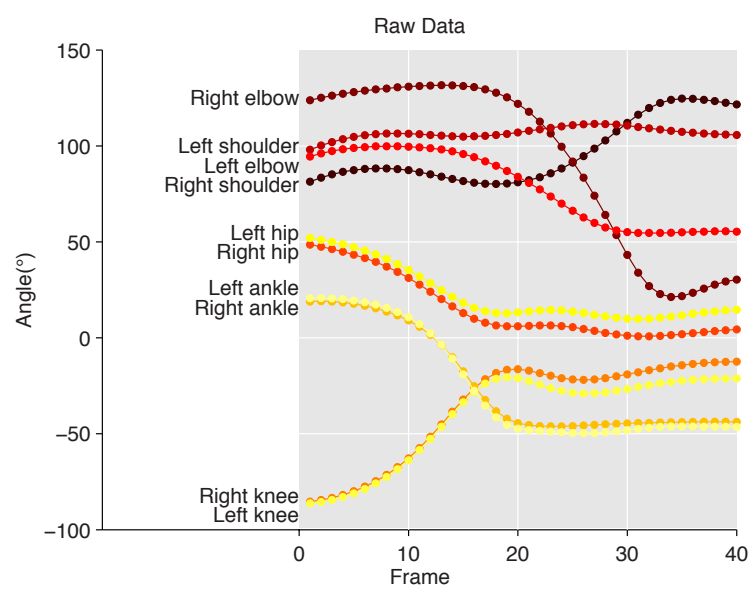

c)

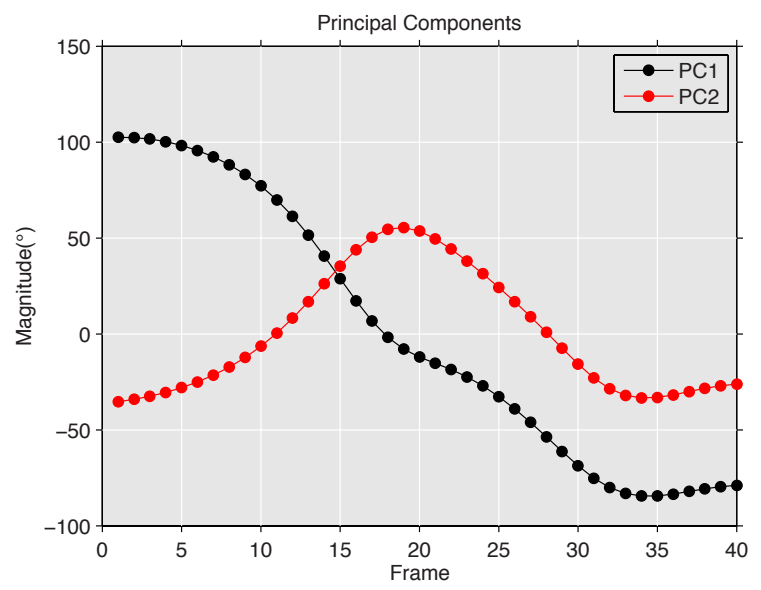

b)

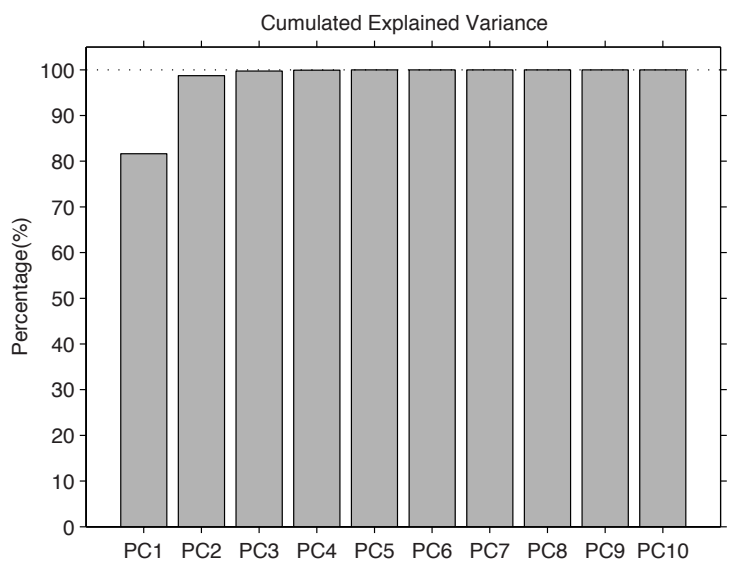

d)

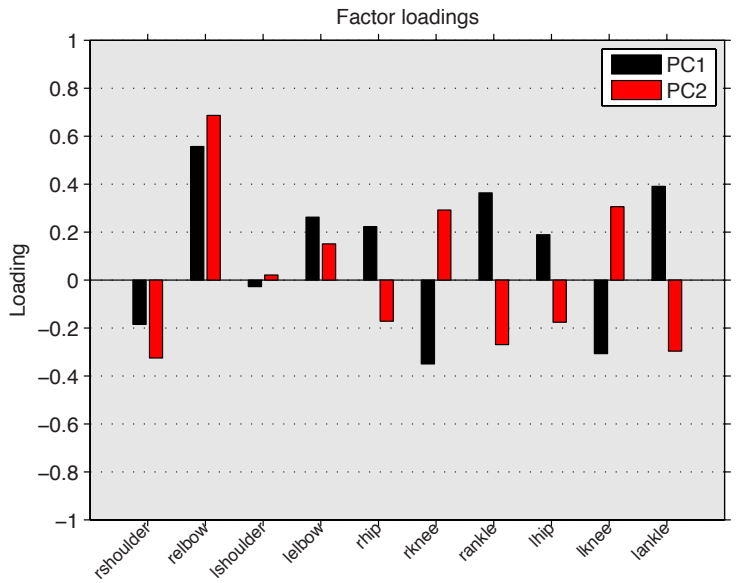

Fig. (4). PCA analysis of a single hoot shot trial. a) raw joint angle curves. b) cumulated explained variance per principal component. c) curves of first two principal components d) principal component loadings for first two principal components.

form whereas the second principal component (PC2) describes a unimodal peak. Inspection of the loadings in Fig. (4d) shows a strong positive loading of the right elbow with $\mathrm{PC} 1$ and negative loadings for the shoulders and the knees and positive loadings for the ankles. Except for the elbow joint angles the loadings are symmetric across the body. This waveform describes the coupled extension of the knees, ankles, and shoulders paired with the flexion in the throwing elbow. The second principal component, explaining much smaller variance, indicates almost equally strong negative loadings for the ankles, hips and the right shoulder and positive loadings for the knees. The right elbow displays the strongest loadings and models the offset of the elbow movement with the flexion occurring after the extension of legs and shoulder. Thus, starting with ten input dimensions the information contained in the data could be reduced to only two dimensions, easing further analysis of the data. See also Daffertshofer et al. [17] for another accessible example.

The eigenvalues $\lambda_{k}$ corresponding to the eigenvectors give a direct estimate of the variance explained by the corresponding principal component. Thus by dividing each eigenvalue by the sum of all eigenvalues one obtains an estimate how much variance is explained through the corresponding principal component $\operatorname{var}_{\text {explained }}=\frac{\lambda_{k}}{\sum_{i=1}^{K} \lambda_{i}}$, as shown in Fig. (3b). Standard software packages like Matlab or GNU R calculate loading, scores, and eigenvalues and are thus readily available without the need for complicated processing of the data. There are no clear-cut conventions about how much variance of the original data must be explained by the principal components for the compression to be sufficient. Typical values range between $80 \%$ and $90 \%$ of the data. Sometimes, higher principal components are actually more interesting as they highlight differences between conditions or actors whereas the first few principal components capture the commonalities across the dataset [17, 42]. Daffertshofer et al. [17] use this "data-driven filter" (p.423) approach to partition the data into deterministic and stochastic components. The deterministic part is captured by the first few main principal components and the smaller components describe the stochastic part. They give an interesting exam- 
ple from walking were the smaller components capture the impact of the foot with the ground [17].

Several insightful applications of PCA are available in the literature. Post, Daffertshofer, and Beek [44] investigated cascade ball juggling during self-paced and fast-paced trials using a PCA approach. Based on their modeling approach of the three balls' flight paths four dimensions were deemed sufficient to model the behavior of the system. The subsequent application of PCA analysis to the ball kinematics corroborated this a-priori modeling. Comparing fast and selfpaced juggling, a switch from four to two main components from the former to the latter condition demonstrated how PCA analysis was able to extract important information about movement organization regarding dimensionality. The authors stressed the importance of studying time series and the spatiotemporal variability, which assists in identification of the most significant events during movement execution without biasing measurement a-priori in contrast to traditional discrete measurements [44]. Chen, Liu, Mayer-Kress, and Newell [30] used PCA for the analysis of the pedalocycling movement. Three to six components extracted by PCA were sufficient for describing $95 \%$ of the variance in the data. The first component accounted for more than half of the variance and its influence increased over the training period. The loading of the components varied between participants. Elipot et al. [45] investigated the underwater gliding motion during swimming start using PCA. They showed that by covarying the shoulder, hip, and knee joint movements swimmers attained a more streamlined posture optimizing underwater gliding velocity [45]. Forner-Corder, Levin, $\mathrm{Li}$, and Swinnen [37] investigated the application of PCA to rhythmical parallel flexion-extension movements of elbows and wrists. The authors calculated the relative phase between the different segments and processed the resulting data with PCA. The identified movement patterning based on PCA matched the a-priori known differences in coordination patterning. E.g. when all four segments were used in an inphase manner a single principle component was sufficient in order to capture data dynamics. These findings lead the authors to conclude that the PCA approach provides valid results. Investigating the limitation of PCA the authors noted that for actions where movements other than strict in-phaseanti phase patterns are prescribed, the resulting principal components structure will be more complex and accordingly more difficult to analyze [37]. Balasubramaniam and Turvey [46] investigated coordination in hula hooping using a nonlinear variant of traditional PCA. Instead of the correlation matrix, the authors used the mutual information between input signals to uncover redundancies in input space [47]. Movements represented by 3D kinematics of the lower limbs joint positions served as input signals. By using the mutual information the authors hoped to protect against nonlinearities and non-stationarities occurring in the input signals [46, p.180]. Other application of PCA include force tasks [48], reaching and grasping [42, 49, 50], muscle activation [51,52], gait [53-56], standing and squatting [40, 57, 58], and stair climbing [59]. Thus, many insightful applications of PCA are available in the literature. However, one has to be aware that apart from different tasks being investigated that there is also a large discrepancy in used data preprocessing methods, which has to be taken into account.
Currently, there is no greed upon standard whether variables should be normalized in time and/or amplitude and how many explained variance is sufficient as these decisions also depend on the specific hypothesis being investigated. A good starting point for further research into principal component analysis is the tutorial by Daffertshofer et al. [17].

As the principal component analysis is based on a linear approach some investigations to extend PCA into the nonlinear domain have been undertaken [60, 61]. Many, of these approaches are based on neural networks schemes in order to estimate the non-linear mapping between input and output variables. However, with neural networks it is somewhat problematic to generalize as the information is distributed across different weights and is not easily accessible [see also $62,63]$. Thus, further work is necessary especially as standard linear PCA is still not used to its full extend.

\section{UNCONTROLLED MANIFOLD ANALYSIS}

The technique of Uncontrolled manifold analysis has been introduced by Scholz and Schöner [64] and is based on ideas from robotic motion-planning. The question underlying uncontrolled manifold analysis is whether movement variability contains certain structure correlated to the task performance. By mapping variability of so-called elementary variables to the variability of a performance variable hidden structural features of action variability are uncovered [64, 65]. Elementary variables can be joint segment angles, joint torques, or some other quantity the researchers believe the system is influencing to control its outcome. These variables span the K degrees of freedom available to the system [65]. The performance or task variables on the other hand are the variables, which are necessary to accomplish a task [65]. Both elementary and performance variables are derived from theoretical considerations. For example, when investigating an upright standing task the elementary variables could be the joint angles and the task variable could be the horizontal position of the centre of mass [66]. In Fig. (5) body configurations for a simple 2D model of upright standing are shown. In Fig. (5a), two different body configurations are depicted which leave the task variable, the position of the centre of mass, unchanged. The elementary variables in this case are the ankle angle, the knee angle, and the hip angle and the neck angle. In Fig. (5b), two body configurations are depicted which yield two different positions of the centre of mass. Thus, variability of the elementary variables can either lead to changes in the performance variable or leaves the performance variable unchanged. The reason why this is possible is a result that more degrees of freedom for the elementary variable compared to the degrees of freedom of the performance variable are available. UCM analysis allows thus to investigate, how the actor deals with this problem.

To perform an uncontrolled manifold analysis of a task, the researcher has to derive a model, which maps the domain of the elementary variables to the domain of the performance variable. A simple example could the task of upright standing, where the centre of mass has to stay within the support area of the feet. To adopt an UCM analysis a geometrical model of the body segments can be used which maps changes in joint segment angles to changes in centre of mass position based on a linked kinematic chain model [67] as the 
joint angles and the positions of the segment centre of masses specify the horizontal position of the body centre of mass (compare Fig. 6) with the latter staying constant. Therefore, the horizontal position of the centre of mass of the body $\mathrm{CoM}_{\mathrm{x}}$ is a function of the joint angles $\operatorname{CoM}_{x}=f\left(\alpha_{\text {ankle }}, \alpha_{\text {knee }}, \alpha_{\text {hip }}, \alpha_{\text {neck }}\right)$.

Assuming the centre of mass of each segment is located at the midpoint of the segment, setting the origin at the tip of the toes, and calculating joint angles as the angle between the body segment and horizontal the following model results:
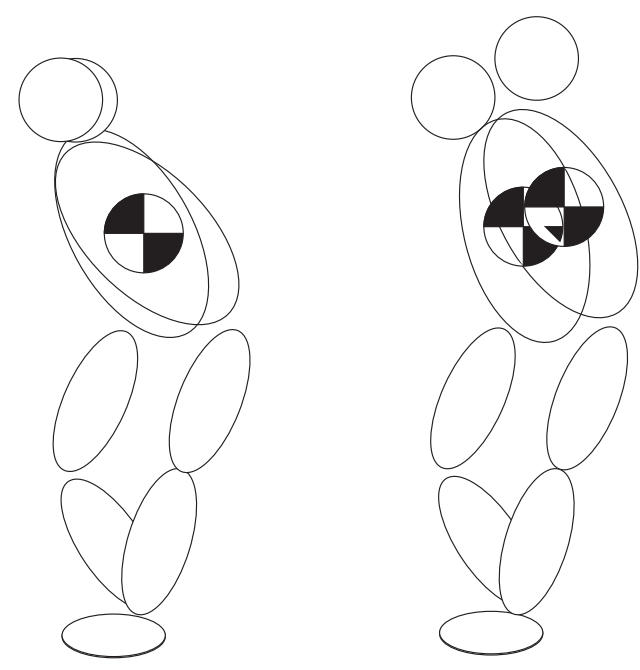

Fig. (5). Body configuration leaving the CoM unchanged (a) and changed (b).

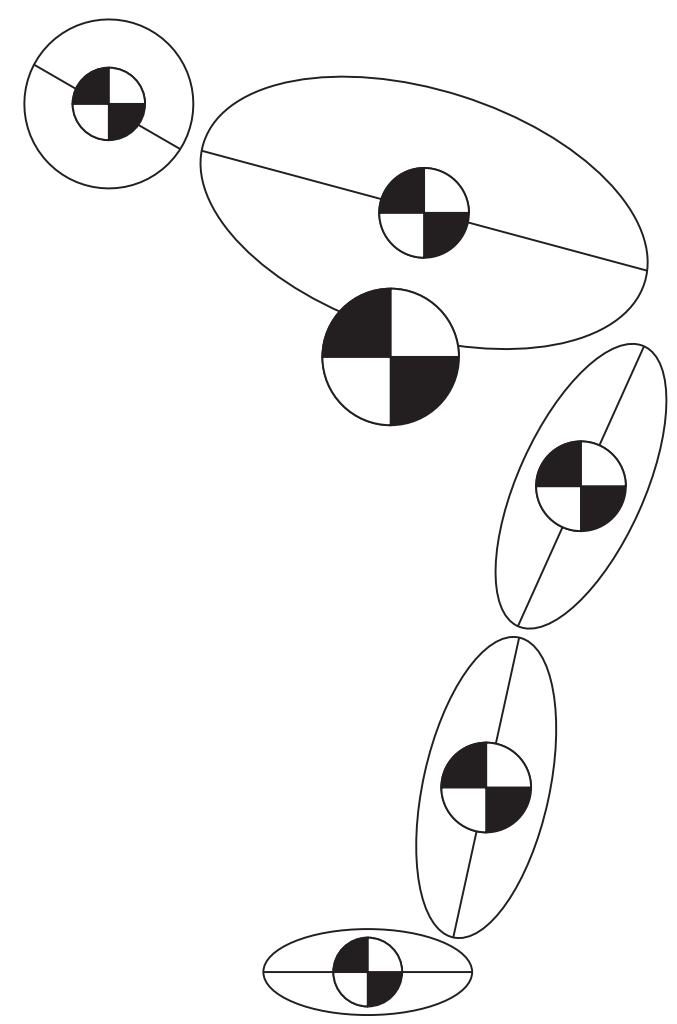

Fig. (6). Simple geometrical model of the body CoM based on individual segment CoM's.

$$
\begin{aligned}
\operatorname{CoM} M_{x}= & 0.5 l_{\text {foot }} m_{\text {foot }}+ \\
& \left(l_{\text {foot }}+0.5 l_{\text {shin }} \cos \left(\alpha_{\text {ankle }}\right)\right) m_{\text {shin }}+ \\
& \left(l_{\text {foot }}+l_{\text {shin }} \cos \left(\alpha_{\text {ankle }}\right)+0.5 l_{\text {thigh }} \cos \left(\alpha_{\text {knee }}\right)\right) m_{\text {thigh }}+ \\
& \left(l_{\text {foot }}+l_{\text {shin }} \cos \left(\alpha_{\text {ankle }}\right)+l_{\text {thigh }} \cos \left(\alpha_{\text {knee }}\right)+0.5 l_{\text {trunk }} \cos \left(\alpha_{\text {hip }}\right)\right) m_{\text {trunk }}+ \\
& \left(l_{\text {foot }}+l_{\text {shin }} \cos \left(\alpha_{\text {ankle }}\right)+l_{\text {thigh }} \cos \left(\alpha_{\text {knee }}\right)+l_{\text {trunk }} \cos \left(\alpha_{\text {hip }}\right)+0.5 l_{\text {head }} \cos \left(\alpha_{\text {neck }}\right)\right) m_{\text {head }}
\end{aligned}
$$

Based on this model formulation, the variability of the elementary task variables can be separated into two orthogonal components. One component describes the variation which alters the value of a to-be-controlled performance variable, the other component leaves the task variable unchanged [64]. All system configurations leaving the performance variable unchanged are collected into a manifold and equally all configurations changing the performance variable are collected into another manifold. The former manifold is called the uncontrolled manifold whereas the latter is the manifold orthogonal to the uncontrolled manifold. The rationale underlying UCM analysis rests on the assumption, that the central nervous system (CNS) should not be concerned with variability which does not change the task outcome and only controls the variability which changes the control variable [64]. The superfluous degrees of freedom can be used by the actor to satisfy additional constraints like energetic efficiency $[35,68]$, which can also be tested using UCM. The separation of the variability of the elementary variable variability is based on the Jacobian of the model, which is the matrix of the first-order partial derivates. In detail, an average trial is formulated and the values for the elementary variables are used to parameterize the Jacobian. For the example of the $\mathrm{CoM}_{\mathrm{x}}$ during simple upright standing the following Jacobian results.

$$
\begin{aligned}
J\left(C o M_{x}\right) & =\left(\begin{array}{lll}
\frac{\partial C o M_{x}}{\partial \alpha_{\text {ankle }}} \frac{\partial C o M_{x}}{\partial \alpha_{\text {knee }}} \frac{\partial C o M_{x}}{\partial \alpha_{\text {hip }}} & \frac{\partial C o M_{x}}{\partial \alpha_{\text {neck }}}
\end{array}\right) \\
\frac{\partial C o M_{x}}{\partial \alpha_{\text {ankle }}} & =-l_{\text {shin }} \sin \left(\bar{\alpha}_{\text {ankle }}\right)\left(0.5 m_{\text {shin }}+m_{\text {thigh }}+m_{\text {trunk }}+m_{\text {head }}\right) \\
\frac{\partial C o M_{x}}{\partial \alpha_{\text {knee }}} & =-l_{\text {thigh }} \sin \left(\bar{\alpha}_{\text {knee }}\right)\left(0.5 m_{\text {thigh }}+m_{\text {trunk }}+m_{\text {head }}\right) \\
\frac{\partial C o M_{x}}{\partial \alpha_{\text {hip }}} & =-l_{\text {trunk }} \sin \left(\bar{\alpha}_{\text {hip }}\right)\left(0.5 m_{\text {trunk }}+m_{\text {head }}\right) \\
\frac{\partial C o M_{x}}{\partial \alpha_{\text {neck }}} & =-l_{\text {head }} \sin \left(\bar{\alpha}_{\text {neck }}\right)\left(0.5 m_{\text {head }}\right)
\end{aligned}
$$

To obtain the uncontrolled manifold, a basis $\left(\varepsilon_{\mathrm{i}}\right)$ spanning the null space of the Jacobian $J\left(\mathrm{CoM}_{x}\right) \varepsilon_{i}=0, \mathrm{i}=1,2, \ldots$, $\mathrm{n}<\mathrm{K}$ and its orthogonal space with $\mathrm{d}=\mathrm{K}-\mathrm{n}$ dimensions is calculated. The former represents the linearized uncontrolled manifold [compare 64 for more details]. If no direct symbolic formula for the mapping can be derived, it is also possible to use a regression model of the elementary variables onto the performance variable to calculate an approximate Jacobian [69]. Once both spaces are obtained the deviations of each trial from the average trial are projected onto these spaces and the magnitude of the projections vectors divided by the dimensions of each space serves as an estimate of the variability. As an average trial must be calculated, UCM 
analysis requires the collection of multiple trials, although there is also the possibility to calculate the UCM from a single trial if one can assume that the task requirements over a certain time do not change [70]. Interpreting the results obtained from UCM, it is possible to test hypotheses about the control of specific performance variables. If the actor is controlling the hypothesized performance variables the normalized projections onto the uncontrolled manifold are larger compared to the projection onto orthogonal space.

The application of UCM analysis is particularly suitable for the application in studies of learning where changes in motor coordination or differences between skill levels can be investigated using this approach. For example, in a study investigating adaptations in reaching under the influence of a force field Yang, Scholz, and Latash [71] showed that the variation of the UCM increased after a learning period. This suggests that the learners acquired the necessary motor skills to take advantage of the UCM [72]. Further applications of UCM analysis include pointing and reaching [71, 73-75], pistol shooting [76], handwriting [77], finger-force production [48], quiet stand [48], and Frisbee throwing [72] have proven its utility for understanding motor control processes. Methodologically, PCA and UCM share some common ground. As UCM investigates the covariation of the elementary variables with regard to the workspace, PCA measures something similar as it is based on the covariation of the variables. However, UCM provides more specific information as the task goal is directly represented in the calculations [78].

Uncontrolled manifold analysis is particular interesting under a non-linear learning paradigm as it provides a different interpretation of movement variability. As movement variability has lost its classical interpretation as noise and error, the UCM framework further supports this view by showing that movement variability can posses inherent structure to ensure task success [79]. This has also some important implications for the interpretations of movement variability and stability. For example, when movement variability is measured using standard methods like range of motion or standard deviations it is actually difficult to make a statement about the stability of movement. The variability could be exclusively within the uncontrolled manifold and thus does not affect task performance at all which challenges traditional notions of movement variability. Taken together, uncontrolled manifold analysis is a useful tool, which can be adapted to different tasks and domains. Through the mapping between process and performance variables UCM provides an elegant way to formulate specific research hypotheses about actions. For a sports scientist, learning uncontrolled manifold analysis will help to gain a much better understanding about the skill underlying sports actions by investigating movement variability. For the interested reader a good starting point is the paper by Scholz and Schöner [64] and the book by Latash [80]. One caveat is again the usage of an average reference trial with the same problems as mentioned above.

\section{CLUSTER ANALYSIS}

Cluster analysis represents a heuristic analysis tool to uncover grouping structure in populations of different ob- jects [81]. Application of cluster analysis is common in various fields of research including biology [82], medicine [83], economics [84]. When an experimenter uses cluster analysis he/she wants to find hidden patterning contained in the data consisting typically of large and complex amounts of data [85]. By summarizing the data through groups, the cluster analysis allows a better and concise understanding of the data. However, cluster analysis sometimes has a somewhat dubious connotation attached to it [85]. In parts this is based on the principles how the clustering algorithms work. Because even when there is no real clustering present in the data the cluster analysis will always group the data into clusters [86]. Thus, the results of each cluster analysis must undergo a validation procedure as otherwise the results may be suspicious [83, 87].

In general, two types of clusters analysis have been used in a movement science context: 1.) Hierarchical cluster analysis and 2.) partitioning or k-means methods [81]. The main difference between them is that partitioning methods will construct a certain number of cluster from the dataset and the number has to be set by the experimenter prior to the analysis. In contrasts, hierarchical cluster analysis will group the data into ever larger clusters and the experimenter has to choose the appropriate number of cluster as part of the analysis result. Thus, sometimes the two methods are used in sequence with first determining the number of cluster using hierarchical cluster analysis as an exploratory step and subsequently refining the clusters using a partitioning method. One serious problem of hierarchical cluster analysis algorithms is their inability to correct an earlier made decision. Thus, once an object is grouped into a cluster this cannot be undone in a later step [88].

The application of cluster analysis demands several processing steps $[88,89]$. First, the desired input variables have to be chosen, and if necessary time and/or range normalized. Each individual trial thereby represents an object for the cluster analysis. Subsequently a distance matrix must be calculated. Central to the application of cluster analysis is the notion of similarity or dissimilarity between objects [88]. The grouping of the dataset by the cluster analysis is based on the specific measure of similarity. Objects, which are similar to each other, are grouped together into one cluster and dissimilar objects are separated into different clusters. Depending on the type and scale of the used variables several different measures are available, including for example Euclidean, Manhatten, and 1-correlation-distance. Setting up an appropriate method to measure similarity between objects is central for an appropriate cluster analysis. For movement data most of the time the Euclidean distance is used [90]. With regard to the 1-correlation distance one can draw a connection to principal component analysis. During each clustering step the correlation between the objects is calculated and the objects are grouped accordingly. This can be interpreted as sort of a hierarchical principal component analysis as the fundamental entity underlying PCA is the variance-covariance matrix [89].

Once a measure method is chosen, a cluster analysis algorithm must be chosen. Again, several different methods are available, each with certain strengths and weaknesses and no simple cookbook approach is possible [81]. Finally, after 
execution of the cluster analysis the obtained results must be validated. Explanation of the different decisions involved in cluster analysis is beyond the scope of the present paper and specialized literature should be consulted [83, 90-92]. The main result of a hierarchical cluster analysis is a dendrogram displaying the grouping structure obtained by the cluster analysis.

In Fig. (7) an example of a dendrogram with synthetic data is shown. For these data the cluster analysis found two different groups from a dataset of 20 objects. The objects contained in each group are depicted by leafs at the bottom of the dendrogram. When objects are connected to each other, the connections indicate that these objects belong to the same cluster. The greater the height between connected objects the greater the difference between them. The dendrogram gives therefore a quick way to assess the results of a hierarchical cluster analysis and should always be included in the results. In Fig. (7) it can be also seen that a hierarchical cluster analysis starts with distinct objects, which are iteratively collected into larger and larger cluster until finally all objects are grouped into a single cluster. The decision for the researcher therefore involves to "cut" the tree at the certain height into different sub-trees. These sub-trees are the resulting clusters. One caveat of cluster analysis is related to the reliability of the results. The cluster analysis algorithm will always group the data into clusters irrespective whether there are any true cluster in the data. Therefore, it is mandatory to always validate the results obtained through cluster analysis. Unfortunately this has not always been the case in the literature. Again, several different validation methods are available in the literature and combining several measures together will give the most robust results [90].

Numerous applications of cluster analysis have been performed in motor control research. Howard and Wilson [91] used a cluster analysis to describe movement patterning in the backstroke swim start in ten participants. Using a body segment model as an input vector, movement postures were grouped according to their similarity in a hierarchical clustering algorithm. The authors were able to identify twentyone model action patterns, which differentiated different starting techniques [91]. Ball and Best [92] investigated weight transfer during the golf swing in 62 participants during ten simulated golf drives. Based on the centre of pressure trajectory, the authors were able to distinguish between different movement strategies used by the participants. Using 3D motion capture, Schöllhorn [93] investigated discus throws in one participant using eight trials in total. The results showed a division of the eight trials into two groups. Jaitner, Mendoza, and Schöllhorn [94] investigated the runup phase in long jumping using a cluster analysis. Based on the obtained clustering the authors were able to determine differences in the execution of the steps prior to the take-off, with great inter-individual differences. Another prominent area where several applications of cluster analysis have been undertaken concerns the study of gait in cerebral palsy patients. O'Byrne, Jenkinson, and O'Brien [95] investigated gait pattern in 146 patients with cerebral palsy using a cluster analysis approach. The resulting grouping was indicative of different walking styles and was used to establish a reliable identification scheme for clinicians. Chow and colleagues [96] investigated the movement patterning during learning of an in-step kick. Using cluster analysis the authors were able

\section{Dendrogram}

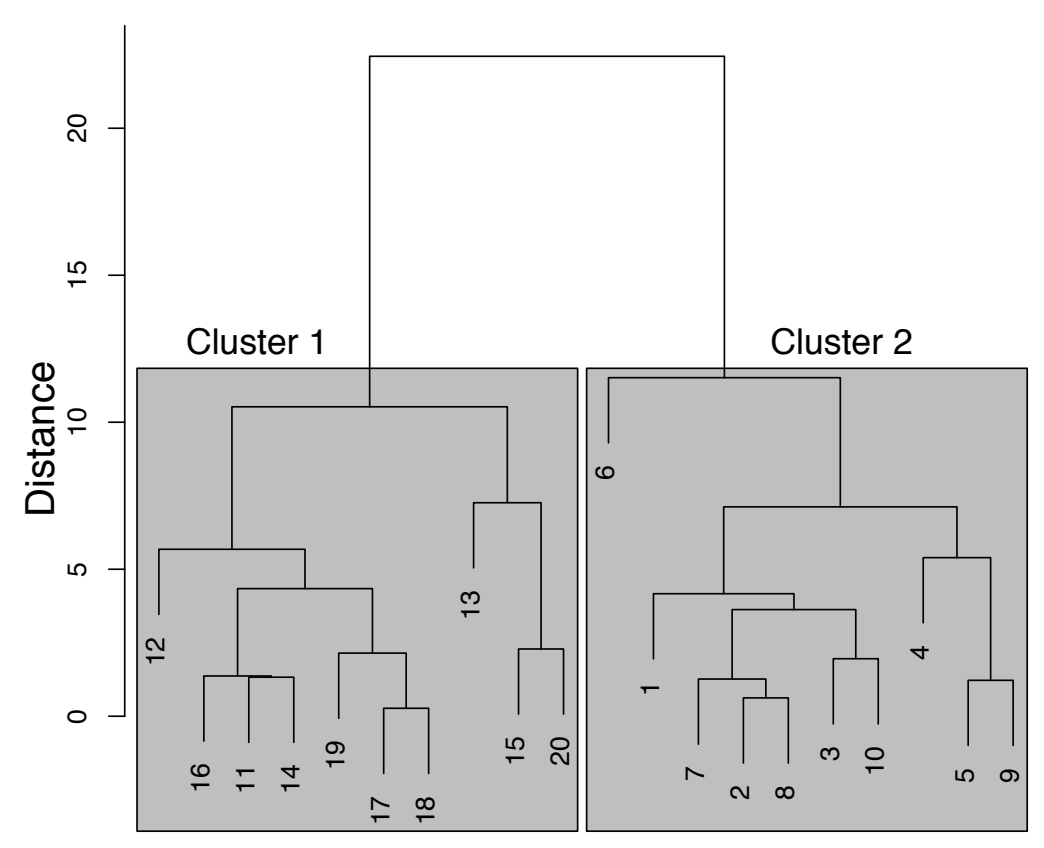

Trial

Fig. (7). Dendrogram of a synthetic dataset obtain using a hierarchical cluster analysis. 
to show that movement patterning did not take a linear route but that the learners should sudden jumps during learning as well as regressions back to earlier movement pattern. Other applications of cluster analysis include basketball [97], handball [98], foot pressure data [99, 100], swimming [101], grip force [102], finger force [103], body sway [104], and finger tremor [105], golf movement patterning [106].

Cluster analysis represents a promising methodological approach that can distinguish between globally similar and different movement patterns. Using a time-continuous approach it is possible to assess timing between different segments together with range of motion information providing interesting insight beyond classical summary statistics. The tutorial by Rein et al. [89] gives further hints about the application of cluster analysis regarding choice of distance and validation methods.

\section{SYNTHESIS AND OUTLOOK}

Several "classical" analysis methods are available which allow researchers in sports sciences to investigate changes occurring during motor learning. The methods presented in the present work allow the analysis of more complex phenomena by going beyond the traditional summary statistics and present a more complete picture of the complex and nonlinear process of motor control and motor learning. At present there is a large body literature movement variability spanning from early important work [8] to more recent investigations [107], thereby demonstrating the importance of movement variability as means for functional action adaptation $[79,108,109]$. The tools described in the present paper enable the researcher to investigate movement variability in more depth and further provide tools to describe the global patterns underlying actions. They assist in identifying collective variables governing the dynamics of the behavior. As these attractors represent a central notion in dynamic systems theory this will also help to extend the scope of dynamical systems theory. Further, the methods presented here make it possible to transfer laboratory paradigms used in classical investigations of dynamical systems theory and apply them to sports movements in the field [5].

Probably the greatest merit will be gained however by not only applying these methods in isolation but by combining the different approaches. For example, uncontrolled manifold analysis is based on the analysis of variance around an average trial. Thus, when datasets are investigated which differ greatly in their waveforms the assumption of a representative average trial becomes problematic. Here cluster analysis could assist by first grouping trials together into clusters and subsequently performing an uncontrolled manifold analysis for each separate cluster. Similar, sometimes using principal component analysis to factor out the important information contained in the data could serve as the first step before a cluster analysis is executed. The challenge for these approaches will be that the researcher has to be able to follow his data through the different processing steps.

The presented methods are particular helpful for sports scientists as they are typically faced with more complex actions compared to standard laboratory tasks, which do not scale easily to complex multi-joint movements $[12,15,21]$. Hereby, in specific the question how to deal with movement variability becomes more and more important. As already explained in the part about UCM, movement variability perse does not say anything about movement stability as this variability can occur within the uncontrolled manifold not affecting the task performance. How is this variability structure when learning a complex movement through the active live span of athlete?

Based on approaches from dynamical systems movement stability has been studied using so-called perturbation paradigms [110-114]. Here the performance of an actor is perturbed through some kind of interference. Observing the dynamics of the behavior, it becomes possible to estimate the stability of the system [115]. However, at present this has been done for only a few movements and much more research on this question seems warranted in order to gain a better understanding about stability of actions. Again, a combination of the different methods introduced here could provide some new insights. For example, when a movement loses stability how does this affect the uncontrolled manifold? When a transition between different movement patterns occurs, how does this affect PCA, clustering and UCM? Is there a relation to the dimensionality of the action as analyzed by PCA prior to movement pattern changes?

The present paper serves only as a first introduction for sports scientists on what methods are used in research and which can be applied to sports researchers. This summary is by no means exhaustive and is mainly due to the preferences of the author and many more methods are available in the literature. However, as the questions about motor learning become more complex so will be the tools to answer these questions. Hopefully the paper created some interest by applied sports scientist and coaches to maybe use some more complex tools for their own data.

\section{CONFLICT OF INTEREST}

The authors confirm that this article content has no conflicts of interest.

\section{ACKNOWLEDGEMENTS}

The author would like to thank to anonymous reviewers for their comments, which significantly improved the quality of the present paper. The connections between PCA, UCM, and cluster analysis were brought to my attention through comments from one of the reviewers.

\section{REFERENCES}

[1] Magill RA. Motor learning: concepts and application. $3^{\text {rd }}$ ed. Dubuque, Iowa: Wm. C. Brown Publishers 1989.

[2] Schmidt RA. A schema theory of discrete motor skill learning. Psychol Rev 1975; 82(4): 225-60.

[3] Schmidt RA, Lee TD. Motor control and learning. $3^{\text {rd }}$ ed. Champaign, IL: Human Kinetics 1999.

[4] Ericsson KA, Lehmann AC. Expert and exceptional performance: evidence of maximal adaptation to task constraints. Annu Rev Psychol 1996; 47: 273-305.

[5] Bartlett $\mathrm{R}$, Wheat J, Robins M. Is movement variability important for sports biomechanists? Sports Biomech 2007; 6(2): 224-43.

[6] Liu Y-T, Mayer-Kress G, Newell KM. Beyond curve fitting: A dynamical systems account of exponential learning in a discrete timing task. J Mot Behav 2003; 35(2): 197-207.

[7] Newell KM, Liu Y-T, Kress GM. Times scales in motor learning and development. Psychol Rev 2001; 108(1): 57-82. 
[8] Turvey MT. Coordination. Am Psychol 1990; 45(8): 938-53.

[9] Haken H, Kelso JAS, Bunz H. A theoretical model of phase transitions in human hand movements. Biol Cybern 1985; 51: 34756.

[10] Kelso JAS. Phase transitions and critical behavior in human bimanual coordination. Am J Physiol Regul Integr Physiol 1984; 246(6):1000-4.

[11] Zanone PG, Kelso JAS. Evolution of behavioral attractors with learning: Nonequilibrium phase transitions. J Exp Psychol Hum Percept Perform 1992; 18(2): 403-21.

[12] Newell KM, Vaillancourt DE. Dimensional change in motor learning. Hum Mov Sci 2001; 20: 695-715.

[13] Newell KM, Liu Y-T, Mayer-Kress G. A dynamical systems interpretation of epigenetic landscapes for infant development. Infant Behav Dev 2003; 26: 229-472.

[14] Newell KM. Change in movement and skill: Learning, retention, and transfer. In: Latash ML, Turvey MT, Eds. Dexterity and its development. Mahwah, N.J.: Erlbaum Associates 1996; pp. 393429.

[15] Rein R, Davids K, Button C. Adaptive and phase transition behavior in performance of discrete multi-articular actions by degenerate neurobiological systems. Exp Brain Res 2010; 201(2): $307-22$.

[16] Button C, Davids K, Schöllhorn W. Coordination profiling of movement systems. In: Davids K, Bennett S, Newell K, Eds. Movement System Variability. Champaign, Illionis: Human Kinetics 2006; p. 133-52.

[17] Daffertshofer A, Lamoth CJC, Meijer OG, Beek PJ. PCA in studying coordination and variability: a tutorial. Clin Biomech 2004; 19(4): 415-28.

[18] Bernstein NA. The co-ordination and regulation of movements. Oxford: Pergamon Press Ltd. 1967.

[19] Sporns O, Edelman GM. Solving Bernstein's problem: A proposal for the development of coordinated movement by selection. Child Dev 1993; 64: 960-81.

[20] Kelso JAS. From Bernstein's physiology of activity to coordination dynamics. In: Latash ML, Ed. Bernstein's traditions in movement studies. Champaign: Human Kinetics 1998; p. 203-19.

[21] Davids K, Button C, Araújo D, Renshaw I, Hristovski R. Movement models from sport provide representative task constraints for studying adaptive behavior in human motor systems. Adapt Behav 2006; 14: 73-94.

[22] Forner-Cordero A, Koopman HJFM, van der Helm FCT. Describing gait as a sequence of states. J Biomech 2006; 39(5): 948-57.

[23] Sidaway B, Heise G, Schoenfelder-Zohdi B. Quantifying the variability of angle-angle plots. J Hum Mov Stud 1995; 29: 181-97.

[24] Mullineaux DR, Bartlett RM, Bennett S. Research design and statistics in biomechanics and motor control. J Sports Sci 2001; 19(10): 739-60.

[25] Hodges NJ, Hays S, Horn RR, Williams AM. Changes in coordination, control and outcome as a result of extended practice on a novel motor skill. Ergonomics 2005; 48: 1672-85.

[26] Chow JY, Davids K, Button C, Koh M. Coordination changes in a discrete multi-articular action as a function of practice. Acta Psychol (Amst) 2008; 127(1): 163-76.

[27] Hallemans A, Aerts P. Effects of visual deprivation on intra-limb coordination during walking in children and adults. Exp Brain Res 2009; 198(1): 95-106.

[28] Crowther RG, Spinks WL, Leicht AS, Sangla K, Quigley F, Golledge J. The influence of a long term exercise program on lower limb movement variability and walking performance in patients with peripheral arterial disease. Hum Mov Sci 2009; 28(4): 494503.

[29] Crowther RG, Spinks WL, Leicht AS, Quigley F, Golledge J. Intralimb coordination variability in peripheral arterial disease. Clin Biomech (Bristol, Avon) 2008; 23(3): 357-64.

[30] Chen H-H, Liu Y-T, Mayer-Kress G, Newell KM. Learning the pedalo locomotion task. J Mot Behav 2005 ;37(3): 247-56.

[31] Page A, Epifanio I. A simple model to analyze the effectiveness of linear time normalization to reduce variability in human movement analysis. Gait Posture 2007; 25: 153-6.

[32] Satern MN, Keller-McNulty S. Use of position-time graphs to compare free throw shooting styles of adult male and female basketball players. J Hum Mov Stud 1992; 22: 13-33
[33] Yu J, Ackland DC, Pandy MG. Shoulder muscle function depends on elbow joint position: an illustration of dynamic coupling in the upper limb. J Biomech 2011; 44(10): 1859-68.

[34] Schöner G, Scholz JP. Analyzing multi-degree-of-freedom movement system based on variance: Uncovering structure vs. extracting correlations. under review.

[35] Latash ML, Scholz JP, Schöner G. Toward a new theory of motor synergies. Motor Control 2007; 11(3): 276-308.

[36] Chau T. A review of analytical techniques for gait data. Part 1: fuzzy, statistical and fractal methods. Gait Posture 2001; 39(1): 4966.

[37] Forner-Cordero A, Levin O, Li Y, Swinnen SP. Principal component analysis of complex multijoint coordinative movements. Biol Cybern 2005; 93(1): 63-78.

[38] Jolliffe I.T. Principal Component Analysis. $2^{\text {nd }}$ ed. New York: Springer 2002.

[39] Stuart M. A geometric approach to principal components analysis Am Stat 1982; 36(4): 365-7.

[40] Alexandrov A, Frolov A, Massion J. Axial synergies during human upper trunk bending. Exp Brain Res 1998; 118(2): 210-20.

[41] Dona G, Preatoni E, Cobelli C, Rodano R, Harrison AJ. Application of functional principal component analysis in race walking: an emerging methodology. Sports Biomech 2009; 8(4): 284-301

[42] Santello M, Flanders M, Soechting JF. Postural hand synergies for tool use. J Neurosci 1998; 18(23): 10105-15.

[43] Haken H. Information and self-organization: a macroscopic approach to complex systems. $3^{\text {rd }}$ ed. Haken H, Eds. Berlin: Springer 2006.

[44] Post AA, Daffertshofer A, Beek PJ. Principal components in threeball cascade juggling. Biol Cybern 2000; 82(2): 143-52.

[45] Elipot M, Hellard P, Taiar R, et al. Analysis of swimmers' velocity during the underwater gliding motion following grab start. $\mathrm{J}$ Biomech 2009; 42(9): 1367-70.

[46] Balasubramaniam R, Turvey MT. Coordination modes in the multisegmental dynamics of hula hooping. Biol Cybern 2004; 90(3): 176-90

[47] Steuer R, Kurths J, Daub CO, Weise J, Selbig J. The mutual information: Detecting and evaluating dependencies between variables. Bioinformatics 2002; 18: 231-40.

[48] Wei Z, Scholz JP, Zatsiorsky VM, Latash ML. What do synergies do? Effects of secondary constraints on multidigit synergies in accurate force-production tasks. J Neurophysiol 2008; 99: 500-13.

[49] Lee D, Corcos DM, Shemmell J, Leurgans S, Hasan Z. Resolving kinematic redundancy in target-reaching movements with and without external constraint. Exp Brain Res 2008; 191(1): 67-81.

[50] Braido $P$, Zhang X. Quantitative analysis of finger motion coordination in hand manipulative and gestic acts. Hum Mov Sci 2004; 22(6): 661-78.

[51] Krishnamoorthy V, Latash ML, Scholz JP, Zatsiorsky VM. Muscle synergies during shifts of the center of pressure by standing persons. Exp Brain Res 2003; 152(3): 281-92.

[52] Perez MA, Nussbaum MA. Principal components analysis as an evaluation and classification tool for lower torso sEMG data. J Biomech 2003; 36(8): 1225-9.

[53] Kienast G, Bachmann D, Steinwender G, Zwick E-B, Saraph V. Determination of gait patterns in children with cerebral palsy using cluster analysis. Gait Posture 1999; 10: 57.

[54] Takei Y, Grasso R, Amorim MA, Berthoz A. Circular trajectory formation during blind locomotion: a test for path integration and motor memory. Exp Brain Res 1997; 115(2): 361-8.

[55] Cheron G, Bouillot E, Dan B, et al. Development of a kinematic coordination pattern in toddler locomotion: planar covariation. Exp Brain Res 2001; 137(3-4): 455-66.

[56] Deluzio KJ, Wyss UP, Zee B, Costigan PA, Sorbie C. Principal component models of knee kinematics and kinetics: Normal vs. pathological gait patterns. Hum Mov Sci 1997; 16(2-3): 201-17.

[57] St-Onge N, Feldman AG. Interjoint coordination in lower limbs during different movements in humans. Exp Brain Res 2003; 148(2): 139-49.

[58] Vernazza-Martin S, Martin N, Massion J. Kinematic synergies and equilibrium control during trunk movement under loaded and unloaded conditions. Exp Brain Res 1999; 128(4): 517-26.

[59] Reid SM, Graham RB, Costigan PA. Differentiation of young and older adult stair climbing gait using principal component analysis. Gait Posture 2010; 31(2): 197-203. 
[60] Saegusa R, Sakano H, Hashimoto S. Nonlinear principal component analysis to preserve the order of principal components. Neurocomputing 2004; 61(0): 57-70.

[61] Karhunen J, Joutsensalo J. Generalizations of principal component analysis, optimization problems, and neural networks. Neural Networks 1995; 8(4): 549-62.

[62] Chau T. A review of analytical techniques for gait data. Part 2: neural network and wavelet methods. Gait Posture 2001:102-20.

[63] Cheng B, Titterington DM. Neural networks: a review from a statistical perspective. Stat Sci 1994; 9(1): 2-54.

[64] Scholz JP, Schöner G. The uncontrolled manifold concept: identifying control variables for a functional task. Exp Brain Res 1999; 126(3): 289-306.

[65] Schöner G, Scholz JP. Analyzing multi-degree-of-freedom movement system based on variance: Uncovering structure vs. extracting correlations. Motor Control 2007; 11(3): 259-75.

[66] Hsu WL, Scholz JP, Schoner G, Jeka JJ, Kiemel T. Control and estimation of posture during quiet stance depends on multijoint coordination. J Neurophysiol 2007; 97(4): 3024-35.

[67] Reisman DS, Scholz JP, Schöner G. Coordination underlying the control of whole body momentum during sit-to-stand. Gait Posture 2002; 15: 45-55.

[68] Latash ML. There is no motor redundancy in human movements. There is motor abundance. Motor Control 2000; 4(3): 259-60.

[69] de Freitas SM, Scholz JP. A comparison of methods for identifying the Jacobian for uncontrolled manifold variance analysis. J Biomech 2010; 43(4): 775-7.

[70] Scholz JP, Kang N, Patterson D, Latash ML. Uncontrolled manifold analysis of single trials during multi-finger force production by persons with and without Down syndrome. Exp Brain Res 2003; 153(1): 45-58.

[71] Yang J-F, Scholz JP, Latash ML. The role of kinematic redundancy in adaptation of reaching. Exp Brain Res 2007; 176(1): 54-69.

[72] Yang JF, Scholz JP. Learning a throwing task is associated with differential changes in the use of motor abundance. Exp Brain Res 2005; 163(2): 137-58.

[73] de Freitas SMSF, Scholz JP, Stehman AJ. Effect of motor planning on use of motor abundance. Neurosci Lett 2007; 417(1): 66-71.

[74] Domkin D, Laczko J, Djupsjobacka M, Jaric S, Latash ML. Joint angle variability in 3D bimanual pointing: uncontrolled manifold analysis. Exp Brain Res 2005; 163(1): 44-57.

[75] Domkin D, Laczko J, Jaric S, Johansson H, Latash ML. Structure of joint variability in bimanual pointing tasks. Exp Brain Res 2002; 143(1): 11-23.

[76] Scholz JP, Schöner G, Latash ML. Identifying the control structure of multijoint coordination during pistol shooting. Exp Brain Res 2000; 135: 382-404.

[77] Latash ML, Danion F, Scholz JF, Zatsiorsky VM, Schöner G. Approaches to analysis of handwriting as a task of coordinating a redundant motor system. Hum Mov Sci 2003; 22(2): 153-71.

[78] Hristovski R, Davids K, Araujo D, Passos P. Constraints-induced emergence of functional novelty in complex neurobiological systems: a basis for creativity in sport. Nonlinear Dynamics Psychol Life Sci 2011; 15(2): 175-206.

[79] Davids K, Glazier P, Araujo D, Bartlett R. Movement systems as dynamical systems: the functional role of variability and its implications for sports medicine. Sports Med 2003; 33(4): 245-60.

[80] Latash ML. Synergy. New York: Oxford University Press 2008.

[81] Everitt BS, Landau S, Leese M. Cluster analysis. $4^{\text {th }}$ ed. London: Arnold 2001.

[82] Handl J, Knowles J, Kell DB. Computational cluster validation in post-genomic data analysis. Bioinform 2005; 21(15): 3201-12.

[83] Blashfield RK. Propositions regarding the use of cluster analysis in clinical research. J Consult Clin Psychol 1980; 48(4): 456-9.

[84] Ketchen DJ Jr, Shook CL. The application of cluster analysis in strategic management research: an analysis and critique. Strategic Manage J 1996; 17(6): 441-58.

[85] Punj G, Stewart DW. Cluster analysis in marketing research: Review and suggestions for application. J Market Res 1983; 20(2): 134-48.

[86] Blashfield RK, Aldenderfer MS. The literature on cluster analysis. Multivariate Behav Res 1978; 13(3): 271-95.

[87] Breckenridge JN. Validating cluster analysis: Consistent replication and symmetry. Multiv Behav Res2000;35(2):261-85.
[88] Kaufmann L, Rousseeuw PJ. Finding groups in data: an introduction to cluster analysis. New York: John Wiley \& Sons Inc. 1990.

[89] Rein R, Button C, Davids K, Summers J. Cluster analysis of movement patterns in multiarticular actions: a tutorial. Motor Control 2010; 14(2): 211-39.

[90] Härdle W, Simar L. Applied Multivariate Statistical Analysis. $2^{\text {nd }}$ ed. Berlin: Springer 2007.

[91] Wilson BD, Howard A, Eds. The use of cluster analysis in movement description and classification of the backstroke swim start. Biomechanics VIII. Proceeding of the Eight International Congress of Biomechanics. Nagoya, Japan 1983.

[92] Ball KA, Best RJ. Different centre of pressure patterns within the golf stroke I: cluster analysis. J Sports Sci 2007; 25(7): 757-70.

[93] Schöllhorn W. Biomechanische Einzellfallanalyse im Diskuswurf: Prozeß- und produktorientierte Technikanalyse mechanischer Energieformen. Frankfurt : Thun 1993.

[94] Jaitner T, Mendoza L, Schöllhorn WI. Analysis of the long jump technique in the transitions from approach to takeoff. Based on time-continuous kinematic data. Eur J Sports Sci 2001; 1(5): 1-11.

[95] O'Byrne JM, Jenkinson A, O'Brien TM. Quantitative analysis and classification of gait patterns in cerebral palsy using a threedimensional motion analyzer. J Child Neurol 1998; 13(3): 101-8.

[96] Chow JY, Davids K, Button C, Rein R. Dynamics of movement patterning in learning a discrete multiarticular action. Motor Control 2008; 12(3): 219-40.

[97] Rein R. Movement coordination in a discrete multi-articular action from a dynamical systems perspective. Ph.D. Dunedin: University of Otago 2007.

[98] Schorer J, Baker J, Fath F, Jaitner T. Identification of interindividual and intraindividual movement patterns in handball players of varying expertise levels. J Mot Behav 2007; 39(5): 40921.

[99] De Cock A, Willems T, Witvrouw E, Vanrenterghem J, De Clerca D. A functional foot type classification with cluster analysis based on plantar pressure distribution during jogging. Gait Posture 2006; 23(3): 339-47.

[100] Giacomozzi C, Martelli F, Nagel A, Schmiegel A, Rosenbaum D. Cluster analysis to classify gait alterations in rheumatoid arthritis using peak pressure curves. Gait Posture 2009; 29(2): 220-4.

[101] Seifert L, Leblanc H, Herault R, et al. Inter-individual variability in the upper-lower limb breaststroke coordination. Hum Mov Sci 2011; 30(3): 550-65

[102] Potter NL, Kent RD, Lindstrom MJ, Lazarus JA. Power and precision grip force control in three-to-five-year-old children: velocity control precedes amplitude control in development. Exp Brain Res 2006; 172(2): 246-60.

[103] Sosnoff JJ, Newell KM. Are age-related increases in force variability due to decrements in strength? Exp Brain Res 2006; 174(1): 86-94.

[104] Lacour M, Barthelemy J, Borel L, et al. Sensory strategies in human postural control before and after unilateral vestibular neurotomy. Exp Brain Res 1997; 115(2): 300-10.

[105] Wharrad HJ, Jefferson D. Distinguishing between physiological and essential tremor using discriminant and cluster analyses of parameters derived from the frequency spectrum. Hum Mov Sci 2000; 19(3): 319-39.

[106] Lames M. Synergetik als Konzept in der Sportmotorik. Sportpsychologie 1992; 3: 12-8.

[107] Newell KM, Corcos DM. Issues in variability and motor control. In: Newell KM, Corcos DM, Eds. Variability and motor control. Champaign IL: Human Kinetics Publishers 1993; p. vi, 510.

[108] Davids K, Glazier P. Deconstructing neurobiological coordination the role of the biomechanics-motor control nexus. Exerc Sport Sci Rev 2010; 38(2): 86-90.

[109] Riley MA, Turvey MT. Variability and determinism in motor behavior. J Mot Behav 2002; 34(2): 99-125.

[110] Cordo PJ, Gurfinkel VS. Motor coordination can be fully understood only by studying complex movements. Prog Brain Res 2004; 143: 29-38.

[111] Belanger M, Patla AE. Corrective responses to perturbation applied during walking in humans. Neurosci Lett 1984; 49(3): 291-5.

[112] Button C, Davids K, Bennett SJ, Taylor MA. Mechanical perturbation of the wrist during one-handed catching. Acta Psychol (Amst) 2000; 105: 9-30. 
[113] Bardy BG, Oullier O, Lagarde J, Stoffregen TA. On perturbation and pattern coexistence in postural coordination dynamics. J Mot Behav 2007; 39(4): 326-36.

[114] Fink PW, Kelso JA, Jirsa VK. Perturbation-induced false starts as a test of the Jirsa-Kelso excitator model. J Mot Behav 2009; 41(2): 147-57.
[115] Scholz JP, Kelso JAS, Schöner G. Nonequilibrium phase transitions in coordinated biological motion: critical slowing down and switching time. Phys Lett A 1987; 123(8): 390-4.

Received: July 29, 2011

Revised: May 25, 2012

Accepted: May 30, 2012

(C) Robert Rein; Licensee Bentham Open.

This is an open access article licensed under the terms of the Creative Commons Attribution Non-Commercial License (http://creativecommons.org/licenses/ by-nc/3.0/) which permits unrestricted, non-commercial use, distribution and reproduction in any medium, provided the work is properly cited. 